\title{
Progressive pseudorheumatoid dysplasia: a case series report
}

\author{
Ziqin Liu^, Xiaobo Chen \\ Department of Endocrinology, Children's Hospital Capital Institute of Pediatrics, Beijing, China \\ Correspondence to: Xiaobo Chen, MD, PhD. Department of Endocrinology, Children's Hospital Capital Institute of Pediatrics, No.2 Ya-Bao Road, \\ Chaoyang District, Beijing 100020, China. Email: 13681094823@163.com.
}

\begin{abstract}
PPRD) is a rare autosomal-recessive, noninflammatory arthropathy. Several cases have been reported worldwide; however, diagnosis remains challenging. Three unrelated children with PPRD were retrospectively studied. All three patients in this study were initially misdiagnosed. The misdiagnoses included juvenile rheumatoid arthritis, myodystrophy and idiopathic short stature. The time from the onset of symptoms to a definitive diagnosis was 3 to 8 years. Clinical signs and radiological phenotypes were analyzed carefully, and they were all consistent with the characteristics of PPRD and noninflammatory polyarticular enlargement. The small joints of both the hands and lower limbs are the most affected. The imaging findings of the patients were flat vertebrae with beak- or bullet-like changes in front of the cone and peripheral metaphysis widening. DNA samples obtained from the family were sequenced to identify the causal gene using whole-exome sequencing (WES). Four Wnt1-inducible signaling pathway protein 3 (WISP3) mutations were verified. c.271delC was not reported previously. The other three mutations, namely, c.136C>T (p. Gln46*), c.667T>G (p. Cys223Gly) and c.589+2T>C, were previously identified. All three patients had a long journey to diagnosis. Early genetic diagnosis can help prevent unnecessary treatments and procedures in patients. Growth hormone is not a good choice for treatment.
\end{abstract}

Keywords: Wnt1-inducible signaling pathway protein 3 (WISP3); progressive pseudorheumatoid dysplasia (PPRD); case report

Submitted Apr 03, 2021. Accepted for publication Jun 10, 2021.

doi: $10.21037 /$ tp-21-152

View this article at: https://dx.doi.org/10.21037/tp-21-152

\section{Introduction}

Progressive pseudorheumatoid dysplasia (PPRD) is a recessive skeletal disorder (MIM 208230). PPRD was first reported by Wynne-Davies (1). Several sporadic cases have been published. The main clinical manifestations are premature degenerative changes of articular cartilage, progressive articular stiffness, articular bulge, deformity, and limited activity. The disease is often asymptomatic in early childhood, with typical symptoms occurring at ages 3 to 8 years (2). The small joints of both the hands and lower limbs are the most affected, and the peripheral joints that are often affected are the small joints of the hand, hip, elbow, knee, wrist, shoulder, ankle and foot. Symptoms include painless swelling of the proximal interphalangeal joints and gait disturbance and difficulty walking due to knee and hip involvement (3). Involvement of the spine is another prominent manifestation of the disease. Early involvement of the spine is often asymptomatic; however, late signs of spinal abnormalities include scoliosis, lumbar lordosis, and thoracic kyphosis deformity. The disorder is associated with mutations in Wnt1-inducible signaling pathway protein 3 (WISP3). WISP3 is a member of the CTGF, CYR61, and NOV (CCN) gene family and can regulate cell proliferation, differentiation and migration (4). In humans with PPRD, Wnt signaling

^ ORCID: 0000-0003-3310-4861. 
may contribute to cartilage degeneration. In 2012, Garcia Segarra et al. (5) reported the largest number of cases of PPRD, i.e., 63 cases, to date. In Turkey, a delayed diagnosis is common, and the average delay in diagnosis is 6 years; in other countries, diagnosis can occur even later in life. Many reports come from orthopedic and rheumatologic departments because of the involvement of lesions in joints. In this report, we present a case series of PPRD and verify a novel WISP3 mutation. We found that growth hormone was not appropriate for patients with PPRD, two of whom had been treated with growth hormone and had worsening symptoms.

We present the following article in accordance with the CARE reporting checklist (available at https://dx.doi. org/10.21037/tp-21-152).

\section{Methods}

\section{Patients}

\section{Case 1}

The patient was an 11-year-old female who, from the age of 5 years, had stiff knees and hip joints, pain, and limited movement and was unable to run. The patient was born naturally at full term and had a normal birth weight and length. She was walking at 16 months. From the age of 4 years, she developed leg pain that became worse while walking but was relieved after rest. She was misdiagnosed with rickets and muscular dystrophy and had been given calcium and vitamin D. Mild deformity of the lower limbs began at age 7 . She was shorter than her peers and was conscious of pain in both knees during exercise; walking was difficult. No dry mouth, nocturia, limb twitching, or morning stiffness was observed. In the previous 2 years, she felt the onset of pain in both knees without inducement. $\mathrm{X}$-ray showed a widened hip space, rough articular surface, suspected bilateral hip ischemia and necrosis, and abnormal epiphysis of the femoral greater trochanter. She was admitted to our department with a main complaint of short stature and knee pain. She did very well in school and had no mental retardation. There was no similar history in the family. She was $133.5 \mathrm{~cm}$ tall $\left(<3^{\text {rd }}\right.$ percentile, $-2 \mathrm{SD}$ ) and weighed $45 \mathrm{~kg}$. She had a spinal hump and slightly lateral spinal bend; both of her knees were slightly varus, with osteopathic enlargement of the distal joints of both hands, knees and elbows. Her spine was stiff. Her C-reactive protein level, rheumatoid factor level and erythrocyte sedimentation rate were normal. The following measurements were recorded: blood calcium, $2.35 \mathrm{mmol} / \mathrm{L}$; phosphorus, $1.7 \mathrm{mmol} / \mathrm{L}$; alkaline phosphatase, $243 \mathrm{IU} / \mathrm{L}$; and IGF-1, $289 \mathrm{ng} / \mathrm{mL}$. A small amount of effusion in the hip joint was found via bilateral hip joint MRI. A vertebral $\mathrm{X}$-ray showed that the cone was generally flat, part of the front edge of the cone was stepped, the intervertebral space was wide in front and narrow in back, and the pedicle was short.

\section{Case 2}

A 12 -year-old male was admitted to the outpatient department because of short stature. He had been healthy for the previous 8 years. He was found to have an abnormal walking style shortly after his $4^{\text {th }}$ birthday. He had pain in both heels that was aggravated when walking long distances. These symptoms worsened with age. No family history of inherited disease was found. He went to a local hospital because of an unsteady gait. He was suspected of having muscular dystrophy and was transferred to a neurologic hospital. The muscle biopsy results were not consistent with muscular dystrophy, and a muscular disease gene panel found no disease-causing genes. Progressive limitations of the hip joint, interphalangeal joint, spine, and knee joint were observed. His experienced heel pain in both feet that was aggravated by fatigue and relieved after rest, and he was misdiagnosed with rheumatoid arthritis at a local hospital; however, he refused to take glucocorticoids and immunosuppressive drugs. He then went to see another doctor, and although his IGF-1 level was $262 \mathrm{ng} / \mathrm{mL}$, he was prescribed growth hormone because of his extremely short stature. One month after the administration of growth hormone, the patient's condition worsened, and he came to our hospital. He was $140 \mathrm{~cm}$ till $\left(<3^{\text {rd }},-1.6 \mathrm{SD}\right)$ and weighed $41 \mathrm{~kg}$. He presented enlargement hand joint, passive posture (Figure 1). He had lordosis of the thoracic cage with mild tenderness, double wrist tenderness with limited movement, enlarged elbows, limited extension, and enlarged knees. His blood calcium level was $2.45 \mathrm{mmol} / \mathrm{L}$, his phosphorus level was $1.5 \mathrm{mmol} / \mathrm{L}$, and his alkaline phosphatase level was 334 IU/L. X-rays (Figure 2) indicated scoliosis, a cone front that looked like the beak of a bird, coxa vara, and an enlarged finger joint and femoral metaphysis.

\section{Case 3}

This patient was a 5-year-old male with slow height growth and unstable gait for 3 years. He broke his left arm in the previous year. The parents did not report a positive family history of hereditary disease. He was admitted to a 

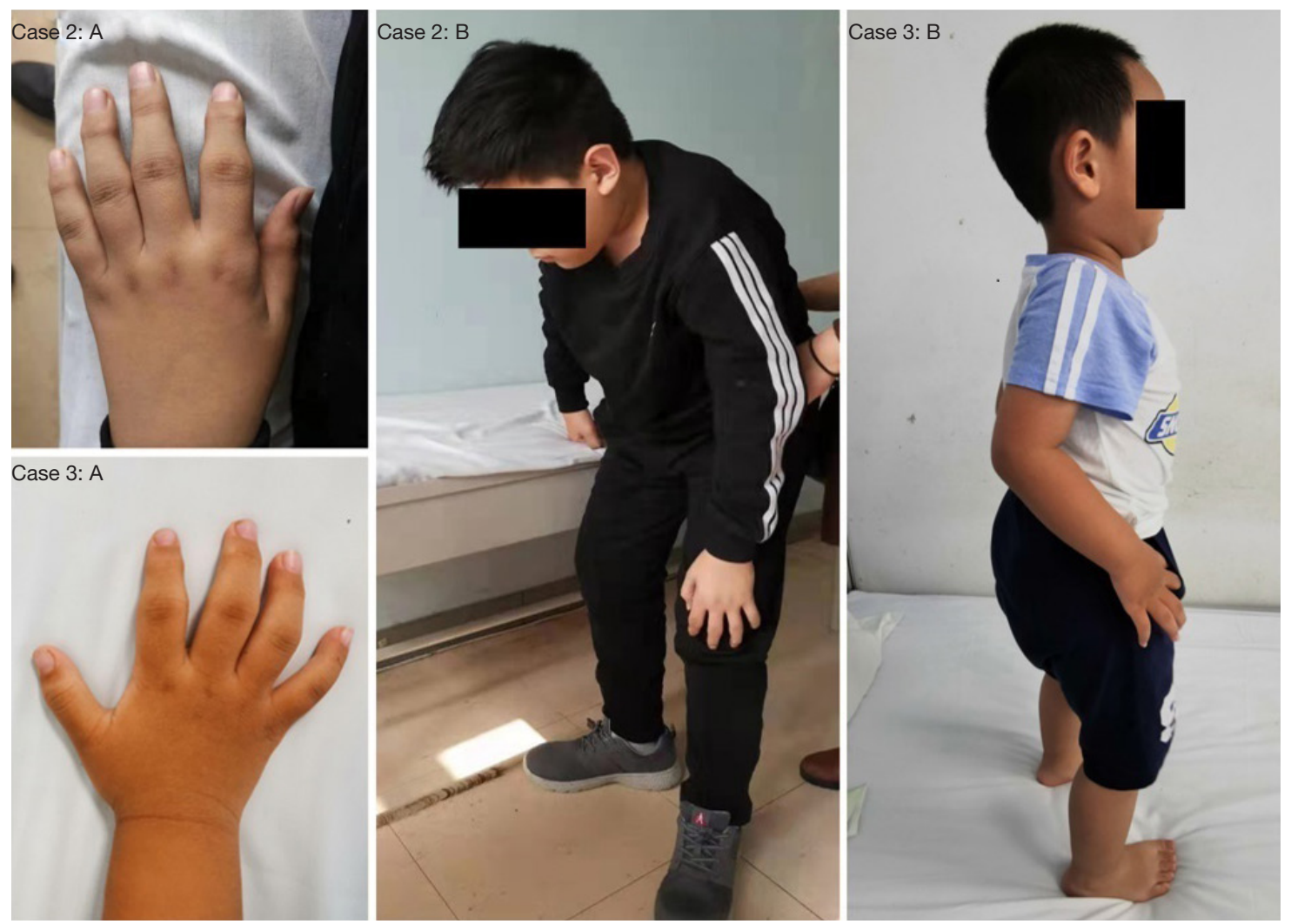

Figure 1 Clinical findings in Case 2 and Case 3. Case 2A and Case 3A: enlargement of the interphalangeal joints, with no swelling; Case 2B and Case 3B: passive posture, stiffness of the spine.

local hospital for the first time 2 years earlier. His alanine aminotransferase (ALT) level was 27 IU/L, his aspartate aminotransferase (AST) level was $58 \mathrm{U} / \mathrm{L}$, his creatine kinase (CK) level was $302 \mathrm{IU} / \mathrm{L}$, and his CK-MB level was $32 \mathrm{U} / \mathrm{L}$, and he was observed without treatment. Two years earlier, he went to a local doctor because of short stature. A pituitary MRI suggested hypophysis dysplasia, which was diagnosed as a partial growth hormone deficiency. He was administered growth hormone treatment for 14 months, and his height increased by $7 \mathrm{~cm}$ but his faltering gait became more serious. One year earlier, the posterior tilt of his buttocks was more pronounced, and his height increased $4 \mathrm{~cm}$ with growth hormone treatment. He did not like walking, preferring to play in bed. His grandmother was 140 $\mathrm{cm}$ tall and had experienced difficulty squatting and getting up since the age of 40 . His developmental milestones were all normal. His height was $99 \mathrm{~cm}\left(<3^{\text {rd }}\right.$ percentile, $\left.-3.3 \mathrm{SD}\right)$, and his weight was $19.8 \mathrm{~kg}\left(50^{\text {th }}\right.$ percentile). He presented a forced posture and was unable to stand upright (Figure 1). He also exhibited a short neck, enlargement of phalangeal joint with joint stiffness, and limitations in bending and squatting. His blood calcium level was $2.25 \mathrm{mmol} / \mathrm{L}$, his phosphorus level was $1.7 \mathrm{mmol} / \mathrm{L}$, his alkaline phosphatase level was $189 \mathrm{IU} / \mathrm{L}$, and his IGF-1 level was $192 \mathrm{ng} / \mathrm{mL}$. $\mathrm{X}$-rays (Figure 2) indicated that the anterior edge of the multiple thoracic and lumbar vertebrae was wedge-shaped and that part of the anterior edge of the cone was pointed. The pelvis was small and narrow, the metaphysis widened, the calcification zone was dense, and the metaphyses of both hands was enlarged.

All procedures performed in studies involving human participants were in accordance with the ethical standards of the institutional and/or national research committee(s) and with the Helsinki Declaration (as revised in 2013). Written informed consent was obtained from the patient.

\section{Molecular studies}

DNA samples obtained from the family were sequenced to identify the causal gene using whole-exome sequencing 

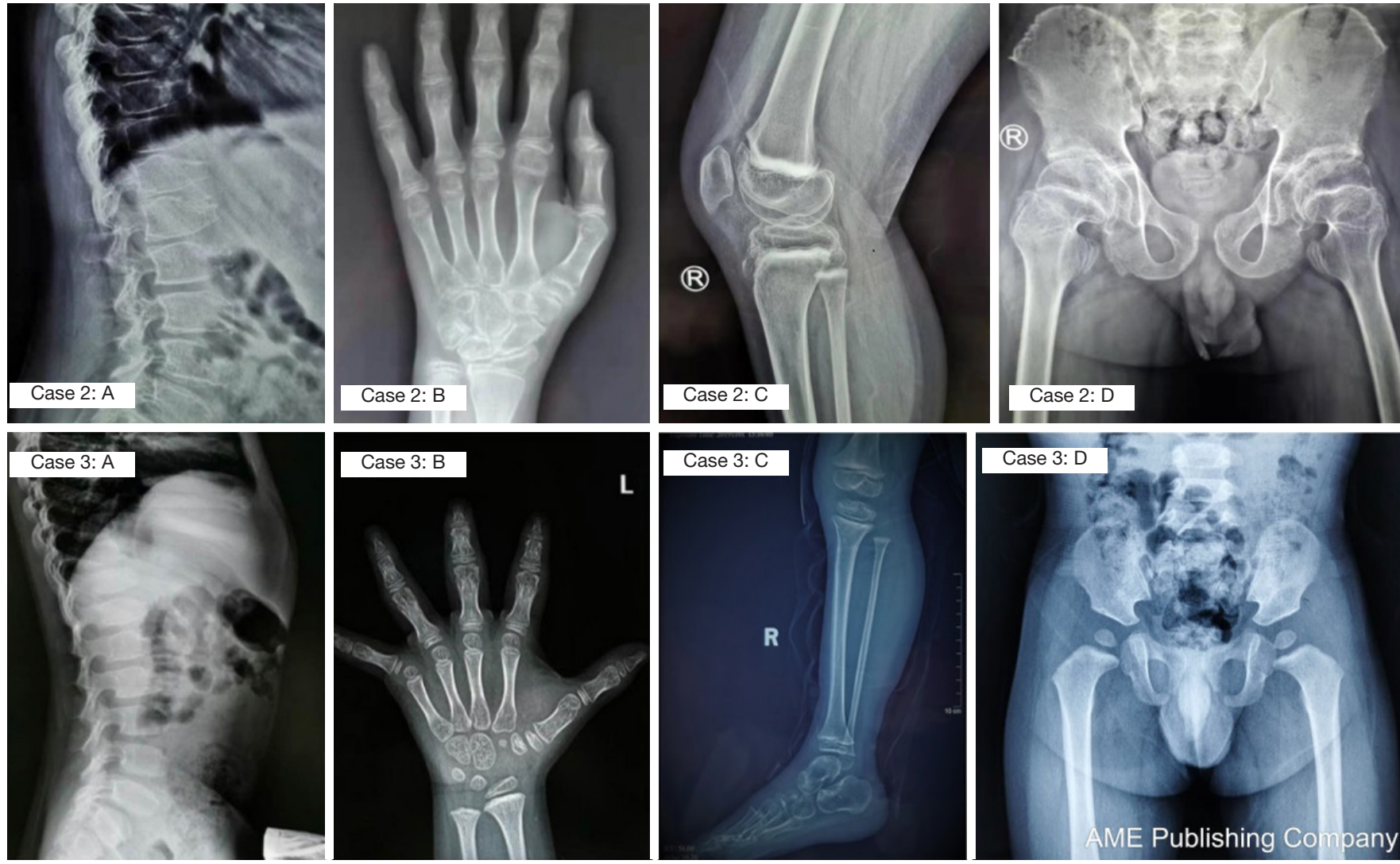

Figure 2 Radiographic findings in Case 2 and Case 3. Case 2A and Case 3A: flattened vertebrae or beaklike changes in the thoracic and lumbar spine. Case 2B and Case 3B: the metaphyses of the fingers on both palms are widened. Case 2C and Case 3C: the metaphysis of the knee is enlarged. Case 2D and Case 3D: A pelvic radiograph showing significant widening of the pubic symphysis space and hypoplasia of epiphysis.

(WES). DNA was isolated from peripheral blood using a DNA Isolation Kit (Bioteke Corporation, AU1802, Wuxi, China). Genomic DNA samples $(1 \mu \mathrm{g})$ were fragmented into 200-300 bp portions using a Covaris Acoustic System (Covaris, Woburn, MA, USA). The DNA fragments were then processed by end repair, A-tailing, adaptor ligation and four-cycle precapture polymerase chain reaction amplification, after which all exons and the 50 bp bases in their adjacent introns were captured with a SeqCap EZ Med Exome Enrichment Kit (Roche, Madison, WI, USA). The DNA library was subjected to postcapture amplification and purification, and then sequencing was performed on an Illumina HiSeq X Ten platform (Illumina, San Diego, CA, USA). The raw data were then filtered and aligned to the human genome reference (hg19) using the BWA Aligner (http://bio-bwa.sourceforge. net/), and variants were identified using NextGene V2.3.4 software (Soft Genetics, LLC, State College PA, USA). The data had a $151.24 \times$ mean read depth, and approximately $97.95 \%$ of the target bases were covered at a $20 \times$ average read depth. The filtered variants were then annotated by using NextGene V2.3.4 and the laboratory's own scripts to obtain related information, including the conservation of nucleotide bases and amino acids, prediction of biological functions, frequency in normal populations (compared with the 1,000 Genomes Project, ExAC, and dbSNP databases and local specific databases), and data from the Human Gene Mutation Database (HGMD), ClinVar and Online Mendelian Inheritance in Man (OMIM). All pathogenic variants were interpreted according to American College of Medical Genetics and Genomics (ACMG) (6) standards and categorized.

\section{Results}

According to a review of the three patients' clinical 


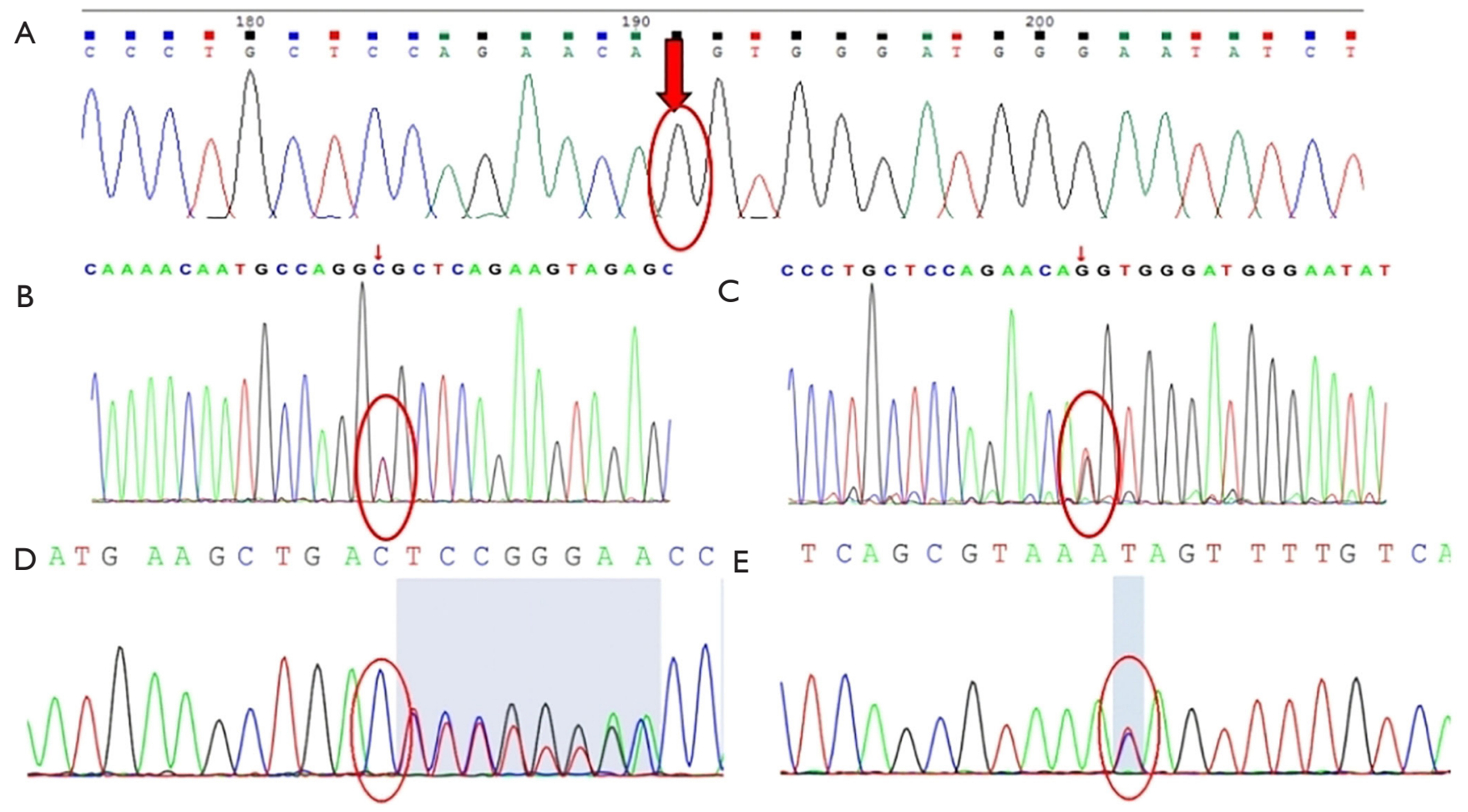

Figure 3 Maps of the WISP3 gene mutation in the three patients. (A) homozygous mutations, c.667T>G carried by patient 1; (B,C) the compound heterozygous mutations, c.667T $>\mathrm{G}$ and c.589+2(IVS4) $\mathrm{T}>\mathrm{C}$ carried by patient 2; (D,E) the compound heterozygous mutations, c.271 delC and c. $136 \mathrm{C}>\mathrm{T}$ carried by patient 3 .

manifestations and X-ray results, all of them presented symptoms and signs of PPRD. The time from the onset of symptoms to a definitive diagnosis was 3 to 8 years. All three children were extremely short. Abnormal gait was the most common presentation. Serum calcium, phosphorus and alkaline phosphatase levels were normal. There were no aberrations in IGF-1 levels. Radiological features were spondyloepiphyseal dysplasia with a short and wide femoral neck, large epiphyses, and narrow joint space at the hips and knees (Figure 2).

Four WISP 3 mutations, which spanned exons 2, intron 3 and exon 4 of WISP3, were identified in the three unrelated patients (Figure 3). Patient 1 was found to be homozygous for the mutation c.667T $>\mathrm{G}$, p. Cys223Gly (PM2+PM3_Verystrong+PP3); her healthy brother had no WISP 3 mutation. Patient 2 was found to have a compound heterozygous mutation (c.667T>G, p. Cys223Gly; c.589+2T>C) $(\mathrm{PM} 2+\mathrm{PM} 3$ Verystrong+PP3, PVS1+PM2+PM3); his healthy sister had no WISP 3 mutation. Patient 3 was found to have a compound heterozygous mutation (c.271delC, p.
Leu91Serfs*14, c.136C >T, p. Gln46*) (PVS1+PM2+PM3, PVS1+PM2+PM3_Strong). The c.136C $>\mathrm{T}$ and c.271delC mutations were in exon 3 , the c. $589+2 \mathrm{~T}>\mathrm{C}$ mutation was in intron 4 , and the c.667T>G mutation was in exon 5. All four variants were predicted to be pathogenic by PolyPhen-2, Mutation Taster and SIFT software. c. $667 \mathrm{~T}>\mathrm{G}$, c. $589+2 \mathrm{~T}>\mathrm{C}$ and c. $136 \mathrm{C}>\mathrm{T}$ were previously reported (7-9). c.271delC is a novel variant of WISP3 and is predicted to cause premature termination of the WISP3 protein.

\section{Discussion}

We employed WES to identify four variants associated with PPRD in three unrelated patients. All three patients in this study were initially misdiagnosed. The misdiagnoses included juvenile rheumatoid arthritis, myodystrophy and idiopathic short stature. The age of onset in all three patients was between 2 and 7 years. The affected children were referred to pediatric neurologists, orthopedic surgeons, or general pediatrics due to nonspecific signs and 
symptoms, specifically muscle weakness and waddling gait. The three patients possessed similar clinical phenotypes. Although all three patients were very short, their IGF1 levels were normal, and their stature was not due to growth hormone deficiency. To our knowledge, the effect of growth hormone therapy in PPRD has not been previously reported; two of the patients had a history of growth hormone treatment, which aggravated the clinical symptoms. Cui et al. reported that WISP3 mutations might inhibit the binding of WISP 3 to IGF-I and thus enhance the effect of IGF-I on chondrocytes (10). Interactions between IGF, IGFBPs, and IGF-IR play important roles in regulating cartilage metabolism, and altering the balance between their levels may result in abnormal maintenance of cartilage tissue (11). These findings may explain the aggravation of patients' illness after growth hormone administration. We do not consider growth hormone treatment appropriate in such patients. Currently, the main treatment for PPRD is supportive care. Physical therapy, walking aids and activity modification could be options for patients with large joint stiffness. Braces can improve scoliosis, and severe deformities of the lower extremities are treated surgically (12).

PPRD is a rare epiphyseal dysplasia. The disease is characterized by progressive bone and joint stiffness, articular enlargement, deformity, and limited mobility and is often clinically misdiagnosed as juvenile rheumatoid arthritis or ankylosing spondylitis. The main reason for delayed diagnosis is the presence of nonspecific and diverse clinical findings and symptoms. Specialists tend to focus more on diseases in their own field and know very little about those in other fields. When clinical symptoms are atypical, misdiagnosis is very common. When they experienced gait instability, none of our patients initially received a spinal $\mathrm{X}$-ray. Spinal $\mathrm{X}$-ray, which is thought to be able to identify PPRD earlier, is recommended. Prenatal morphogenesis or skeletal growth appears undisturbed (4). Changes in bone appear gradually, which is why PPRD is often misdiagnosed clinically as rheumatoid arthritis. Imaging gives clinical indications when symptoms become apparent. This disease should be of concern to pediatric rheumatologists, endocrinologists, orthopedic doctors and health care practitioners.

WISP3 is a member of the CCN gene family. Members of this family encode cysteine-rich secreted proteins that play roles in cell growth and differentiation (13). WISP 3 encodes a 354 amino acid protein that maps to chromosome 6q21-22 and contains a signaling peptide
(SP) and four conserved cysteine-rich domains. In vitro studies have shown that WISP3 regulates the expression of collagen type II, the transcription factor SOX9 and aggrecan in chondrocytes; promotes superoxide dismutase activity $(14,15)$; inhibits cell proliferation and viability; and promotes the differentiation of human chondrocyte precursor cells, thus affecting cartilage homeostasis $(16,17)$. The first report indicating that WISP 3 mutations cause PPRD, which was published in 1999, showed that these mutations interfere with normal cartilage homeostasis, leading to precocious joint degeneration (4). More than 70 loss-of-function variants in WISP 3 have been reported to date $(5,18,19)$. There is no clear genotype-phenotype correlation (20).

c. $271 \mathrm{delC}$ is a novel variant of WISP 3 that results in amino acid changes (p. L91SFS *14, code-shifting variation, terminates after 14 bases); thus, we speculated that it is a pathogenic mutation. This variant has not been reported in the HGMD Pro database or ClinVar. c.667T>G has been reported in many cases in Chinese families $(3,7,19)$, but more evidence is needed to support whether it is a hotspot mutation in Chinese patients. c. 136C $>\mathrm{T}$ has been reported in 3 Chinese families $(9,14,19)$ in addition to the patient in our study but has not been reported in any other countries. c. $589+2 \mathrm{~T}>\mathrm{C}$ was reported in a Chinese family (8). The c. $156 \mathrm{C}>\mathrm{A}$ mutation is frequently found in patients in countries such as Turkey, Lebanon, Syria, Italy, France, and India, but the most common mutations in India are c.1010G > A and c. $233 \mathrm{G}>\mathrm{A}$ (14). Although there have been no reports of hotspot mutations of WISP3, the frequencies of different mutations vary geographically. Given that early diagnosis is difficult and prevents unnecessary examinations and treatment attempts, molecular diagnosis is strongly recommended. Early genetic diagnosis can spare patients from unnecessary treatments and procedures.

In conclusion, a novel variant of WISP3 (c.271delC) that causes PPRD was identified in our study. The diagnosis of PPRD is a challenge for doctors. A focus on bone $\mathrm{X}$-rays, especially spinal $\mathrm{X}$-rays, is conducive to early diagnosis. Growth hormone is not a good choice for treatment.

\section{Acknowledgments}

Funding: None.

\section{Footnote}

Reporting Checklist: The authors have completed the 
CARE reporting checklist. Available at https://dx.doi. org/10.21037/tp-21-152

Conflicts of Interest: Both authors have completed the ICMJE uniform disclosure form (available at https://dx.doi. org/10.21037/tp-21-152). The authors have no conflicts of interest to declare.

Ethical Statement: The authors are accountable for all aspects of the work in ensuring that questions related to the accuracy or integrity of any part of the work are appropriately investigated and resolved. All procedures performed in studies involving human participants were in accordance with the ethical standards of the institutional and/or national research committee(s) and with the Helsinki Declaration (as revised in 2013). Written informed consent was obtained from the patient.

Open Access Statement: This is an Open Access article distributed in accordance with the Creative Commons Attribution-NonCommercial-NoDerivs 4.0 International License (CC BY-NC-ND 4.0), which permits the noncommercial replication and distribution of the article with the strict proviso that no changes or edits are made and the original work is properly cited (including links to both the formal publication through the relevant DOI and the license). See: https://creativecommons.org/licenses/by-nc-nd/4.0/.

\section{References}

1. Wynne-Davies R, Hall C, Ansell BM. Spondylo-epiphysial dysplasia tarda with progressive arthropathy. A "new" disorder of autosomal recessive inheritance. J Bone Joint Surg Br 1982;64:442-5.

2. Montané LS, Marín OR, Rivera-Pedroza CI, et al. Early severe scoliosis in a patient with atypical progressive pseudorheumatoid dysplasia (PPD): Identification of two WISP3 mutations, one previously unreported. Am J Med Genet A 2016;170:1595-9.

3. Ye J, Zhang HW, Qiu WJ, et al. Patients with progressive pseudorheumatoid dysplasia: From clinical diagnosis to molecular studies. Mol Med Rep 2012;5:190-5.

4. Hurvitz JR, Suwairi WM, Van Hul W, et al. Mutations in the CCN gene family member WISP3 cause progressive pseudorheumatoid dysplasia. Nat Genet 1999;23:94-8.

5. Garcia Segarra N, Mittaz L, Campos-Xavier AB, et al. The diagnostic challenge of progressive pseudorheumatoid dysplasia (PPRD): A review of clinical features, radiographic features, and WISP3 mutations in 63 affected individuals. Am J Med Genet Part C Semin Med Genet 2012;160C:217-29.

6. Richards S, Aziz N, Bale S, et al. Standards and guidelines for the interpretation of sequence variants: a joint consensus recommendation of the American College of Medical Genetics and Genomics and the Association for Molecular Pathology. Genet Med 2015;17:405-24.

7. Yu Y, Hu M, Xing X, et al. Identification of a mutation in the WISP3 gene in three unrelated families with progressive pseudorheumatoid dysplasia. Mol Med Rep 2015;12:419-25.

8. Ehl S, Uhl M, Berner R, et al. Clinical, radiographic and genetic diagnosis of progressive pseudorheumatoid dysplasia in a patient with severe polyarthropathy. Rheumatol Int 2004;24:53-6.

9. Hu WW, Zhang ZL. Identification of WISP3 gene mutation in four families with progressive pseudorheumatoid dysplasia. Chinese Journal of Osteoporosis and Bone Mineral Research 2018;11:224-32.

10. Cui RR, Huang J, Yi L, et al. WISP3 suppresses insulinlike growth factor signaling in human chondrocytes. Mol Cell Endocrinol 2007;279:1-8.

11. Matsumoto T, Tsurumoto T, Goldring MB, et al. Differential effects of IGF-binding proteins, IGFBP-3 and IGFBP-5, on IGF-I action and binding to cell membranes of immortalized human chondrocytes. J Endocrinol 2000;166:29-37.

12. Bhavani GS, Shah H, Shukla A, et al. Progressive Pseudorheumatoid Dysplasia. University of Washington, Seattle: Gene Reviews 2015:1993-2021.

13. Temiz F, Ozbek MN, Kotan D, et al. A homozygous recurring mutation in WISP3 causing progressive pseudorheumatoid arthropathy. J Pediatr Endocrinol Metab 2011;24:105-8.

14. Torreggiani S, Torcoletti M, Campos-Xavier B, et al. Progressive pseudorheumatoid dysplasia: a rare childhood disease. Rheumatol Int 2019;39:441-52.

15. Sen M, Cheng YH, Goldring MB, et al. WISP3-dependent regulation of type II collagen and aggrecan production in chondrocytes. Arthritis Rheum 2004;50:488-97.

16. Miller DS, Sen M. Potential role of WISP3 (CCN6) in regulating the accumulation of reactive oxygen species. Biochem Biophys Res Commun 2007;355:156-61.

17. Davis L, Chen Y, Sen M. WISP-3 functions as a ligand and promotes superoxide dismutase activity. Biochem Biophys Res Commun 2006;342:259-65.

18. Delague V, Chouery E, Corbani S, et al. Molecular study 
of WISP3 in nine families originating from the middleeast and presenting with progressive pseudorheumatoid dysplasia: identification of two novel mutations, and description of a founder effect. Am J Med Genet A 2005;138A:118-26.

19. Sailani MR, Chappell J, Jingga I, et al. WISP3 mutation associated with pseudorheumatoid dysplasia. Cold Spring Harb Mol Case Stud 2018;4:a001990.

20. Pode-Shakked B, Vivante A, Barel O, et al. Progressive Pseudorheumatoid Dysplasia resolved by whole exome sequencing: a novel mutation in WISP3 and review of the literature. BMC Med Genet 2019;20:53.
Cite this article as: Liu Z, Chen X. Progressive pseudorheumatoid dysplasia: a case series report. Transl Pediatr 2021;10(7):1932-1939. doi: 10.21037/tp-21-152 